\title{
A Node Model Capturing Turning Lane Capacity and Physical Queuing for the Dynamic Network Loading Problem
}

\author{
Mingxia Gao \\ School of Traffic and Transportation, Lanzhou Jiaotong University, Lanzhou 730070, China \\ Correspondence should be addressed to Mingxia Gao, mxgao@mail.lzjtu.cn
}

Received 8 May 2012; Revised 30 August 2012; Accepted 30 August 2012

Academic Editor: Wuhong Wang

Copyright (c) 2012 Mingxia Gao. This is an open access article distributed under the Creative Commons Attribution License, which permits unrestricted use, distribution, and reproduction in any medium, provided the original work is properly cited.

An analytical dynamic node-based model is proposed to represent flows on a traffic network and to be utilized as an integral part of a dynamic network loading (DNL) process by solving a continuous DNL problem. The proposed model formulation has an integrate base to be structured with a link load computing component, where physical queuing and its influence were explicitly taken into account by dividing a link into two parts: running part and queuing part. The solution to the model is obtained by a hybridization algorithm of simulation and analytical approach, where an iteration process is conducted to update time-dependent network flow conditions after a reasonable discretization of the problem. The performance of the proposed model, as a DNL model, is tested on a sample network. It is seen that the proposed model provides consistent approximations to link flow dynamics. The dynamic node model proposed in this paper is unique in that it explicitly models directional queue in each turning lane and the First-In-First-Out (FIFO) rule at lane level rather than link level is pursued.

\section{Introduction}

The dynamic network loading (DNL) problem is the reproduction of variable link performances and network flow conditions by considering nodal and exiting flow characteristics [1-4] or by explicitly considering the flow propagation and the provision of real time pathlink information [5-7], given path flows and link-performance functions. In the past two decades, DNL has been extensively studied owing to the needs of simulating urban traffic and solving dynamic traffic assignment (DTA) problems. The variation in model structure is heavily dependent on both assumptions made to obtain a solution for the problem, that is, the discretization dimension, queuing, and the criteria that affect the computation of link loads and path-link traveling times. One way to categorize the different approaches is discrete-flow 
models and continuous-flow models. Discrete-flow models, also referred to as simulations, are usually classified into microscopic simulations and macroscopic simulations. In this paper we concentrate on continuous-flow models which are also referred as "analytic" or "macroscopic" models, and we do so for the usual reasons: low number of parameters to be calibrated, good computational performance, mathematical tractability. Microsimulations are time-based, meaning that individual vehicles are moved in short-time intervals $(0.1-1 \mathrm{~s})$ according to car following and other traffic behavior models [8]. The main assumption that is made during the construction of a model to solve DNL problem is on queuing and can be divided into point queuing and physical queuing. Considering the queuing assumption, the models of DNL can be further classified as link based, which vary with the adopted performance function such as link exit function and travel-time-function and node based. Although the performance-function-adopted-link-based modeling framework has been widely used for the analytical formulation of the DNL problem, little research has been conducted to explore their capabilities of reproducing realistic flow dynamics [9-11].

The term node based is generally used for models that explicitly consider the flow splitting rates which are the proportions of traffic leaving a node, assigned to each exiting link, and has been originated from the pioneering proposition of the cell-based traffic flow model in [12]: the cell transmission model (CTM). Node models can be classified as follows according to how link interactions are modeled: (1) competition-free nodes: only the flow conservation law is obeyed at such nodes. The competition-free node model is often seen in the analytical DTA research [13]. (2) Uncontrolled competition nodes: traffic from different incoming links and/or heading to different outgoing links would have to compete against each other for the limit capacities [14]. A typical example is freeway junctions (on- and offramps) without metering facilities. (3) Controlled competition nodes: the competition among different traffic streams is managed by a controlled logic, such as signalized intersections. No matter if controlled or uncontrolled competition nodes, the local demand and supply flow concept are utilized to put up an unifying framework for the modeling of intersections simultaneously with the imposition of boundary conditions to various network flow models such as first-order wave models in [12,15], second-order wave models in [16], models that explicitly incorporate the spatial queuing effect [9], and link performance models [17]. Most of the aforementioned node-based models are proposed to overcome the deficiencies of linkbased models and to be utilized in network traffic control and management applications, where merges and diverges excessively break down the stability of traffic flow.

However, some details that are really important and strongly influence loading results have not got satisfactory representation and enough attention. For example, most node models deal with flow propagation through nodes by distributing local demand of upstream links to downstream links constrained by prevailing supply, where the prevailing demand of a link depends on flows waiting to exit and the capacity of the link rather than the capacity of turning lanes. Queuing on an upstream link is treated as a whole rather than capturing turning directions of vehicles or directional lanes they belonging to. To satisfy FIFO at link level, such models are liable to cause unrealistic description of flow dynamics. For example, rightturning vehicles have to wait even if its downstream link has enough supply just because left-turning vehicles cannot cross the intersection for lacking of downstream supply, whether these vehicles (right turning and left turning) are in the same directional turning lane. In real traffic conditions, as pointed in HCM, flows propagating through an intersection are limited by the capacity of directional entry lanes, and drivers generally choose a turning lane according to their turning directions when approaching an intersection, which leads to several directional queues with different flow dynamics even on the same link. 
Being cognizant of the insufficiency of link-based models in reproducing actual traffic dynamics and the motivation for node-based models, we have proposed an analytical dynamic node-based model that explicitly considers the influence of directional turning lane and physical queuing in different lanes. In our model, queues in each turning lane are explicitly modeled and FIFO at lane level is pursued. We have chosen to solve the continuous DNL problem analytically with our proposed methodology rather than formulating a theoretical high-order wave model for a node. The proposed node model has an integrate base to be structured with a link load computing component. The constraints of link dynamics, flow conservation, flow propagation, and boundary conditions are considered both in nodal rules and the link model. We obtained the solution of the model by coding a simulation-based hybridization algorithm after designing a discrete version of the problem.

This paper is organized as follows. In Section 2, model description, including the link model and the node model formulation is given. The solution procedure is explained in Section 3. Section 4 holds the numerical results of the solution method that is employed to solve the DNL problem on a sample network. Findings and discussions based on the results obtained conclude this paper as the final section.

\section{Model Description}

Given path flow and link performance functions, the CDNLP consists of determining timedependant network flow conditions such as link travel times, link inflows, and link outflows. In the loading procedure, the modeling of traffic flow has two major facets: the representation of traffic dynamics on a link (homogeneous road segment) and on a node (boundary of several links, intersection). We propose an approach that evolves a link model with a node model, where a link is divided into two parts: the running part and the queuing part. The partial length of the running part is dependent on the prevailing traffic condition. Flow dynamics on the running part are described by a Travel Time Function (TTF-) based link model, and flow propagation through intersections is represented with a node model that describes directional queuing in different turning lanes explicitly. Without loss of generality, all links are assumed to have exclusive turning lanes for each turning direction at intersections. Time-dependent exit flow of a directional lane rather than link exit flow is modeled and calculated as the minimum value of prevailing demand and supply of the lane. For links holding mixed turning lanes, exit flow from mixed lanes involves distributing lane demand to downstream links, which can be dealt with using methods adopted in previous node models $[18,19]$ by taking a mixed lane as a link.

Some basic notations and variables are given first. The physical traffic network can be represented by a directed network $G=(N, A)$, where $N$ is the set of nodes, and $A$ is the set of links. In the following, the index $r$ denotes an origin node, the index $s$ denotes a destination node, the index $a$ denotes a link, the index $p$ denotes a path between the origin $(O)$ and the destination $(D)$, and the index $a a^{+}$denotes a directional turning lane which connects link $a$ and its downstream link $a^{+}$. An $O-D$ pair $(r, s)$ is designated, where $O \subset S, D \subset S, r \in O$, $s \in D, r s \in R$, and $R \subset(O * D)$. The $\subset$ of paths between an $O-D$ pair $(r, s)$ is denoted by $p_{r s}$.

\subsection{Link Model}

Link models for the DNL problems enable the specification of flow dynamics on a link in three ways: bottleneck models, whole link function models, and hydrodynamic models. In 
bottleneck-type models, vehicles always move along a link at free-flow speed before they arrive at the exit node, where they form a FIFO queue if the outflow rate they induce exceeds the maximum discharge rate (bottleneck capacity) of the link [19]. In whole-link function models, a performance function (Travel-Time-based Function or Link Exit Function) is adopted to describe flow dynamics taking the whole link as homogeneous [20]. While hydrodynamic models view traffic as a continuous fluid represented by density, speed, and flowrate; they are also known as kinematic wave (KW) models because their solutions can be categorized by combinations of kinematic waves in any of the three quantities [21]. We follow the approach of bottleneck models because flow dynamics of vehicles running on homogeneous segments and of those waiting in a queue for exiting should be treated differently. Also, some improvements are made to model congestions on a link segment and to capture the effect of physical queuing. In our approach, a link is divided into two parts: the running part and the queuing part. The prevailing partial length of the running part of link $a$ (denoted with $L_{a}^{\prime}(t)$ ) depends on the number of vehicles on the link and is calculated as below:

$$
L_{a}^{\prime}(t)=L_{a} \times\left(1-\frac{x q_{a a^{+}}^{*}(t)}{x r_{a}(t)+x q_{a}(t)}\right)
$$

where $x q_{a a^{+}}^{*}(t)$ is the number of vehicles in a turning lane of link $a$ with longest queue at time $t ; x r_{a}(t)$ and $x q_{a}(t)$ denote the number of vehicles on the running part and the queuing part of link $a$ at time $t$.

The traversal time on the running part of link $a$ experienced by vehicles entering at time $t$ (denoted with $d r_{a}(t)$ ) is a function of link volume as given in (2.2):

$$
d r_{a}(t)=\frac{L_{a}^{\prime}(t)}{v_{a}(t)}
$$

where $v_{a}(t)$ denotes the travel speed for vehicles entering link $a$ at time $t$ and is calculated with a modified Green-Shields equation [22] as given by (2.3):

$$
v_{a}(t)=v_{a, \min }+\left(v_{a, f}-v_{a, \min }\right)\left(1-\frac{k_{a}(t)}{k_{a, j}}\right)
$$

where $v_{a, \min }, v_{a, f}, k_{a, j}$ denote the minimum speed, free-flow speed, and jam density of link $a$, respectively. The prevailing density of the running part of link $a$ at time $t$ (denoted with $\left.k_{a}(t)\right)$ is determined by the following equation:

$$
k_{a}(t)=\frac{x r_{a}(t)}{L_{a}^{\prime}(t) \cdot \operatorname{lan}_{a}}
$$

where $\operatorname{lan}_{a}$ denotes the lane number of link $a$.

The path-specific exit flow of the running part of link $a$ is determined by (2.5):

$$
v r_{r s p}^{a}\left(t+d r_{a}(t)\right)=\frac{u r_{r s p}^{a}(t)}{1+d r_{a}(t)-d r_{a}(t-1)}, \quad \forall r, s, t, a, \forall p \in p_{r s}
$$


where $u r_{r s p}^{a}(t)$ and $v r_{r s p}^{a}(t)$ denote entering flow and exit flow of the running part of link $a$ of $r_{s} O-D$ pair on path $p$ at time $t$. $u r_{r s p}^{a}(t)$ can be determined beforehand as below:

$$
u r_{r s p}^{a}(t)=\left\{\begin{array}{ll}
f_{r s p}(t), & a \text { is the first link on path } p, \\
v q_{r s p}^{\left(a_{p}^{-}\right) a}(t), & \text { otherwise, }
\end{array} a, a^{-} \in p,\right.
$$

where $a_{p}^{-}$is the link that precedes link $a$ on path $p ; v q_{r s p}^{a_{p}^{-} a}(t)$ is the exit flow from the queuing part of link $a^{-}$in turning lane $a^{-} a$ of $r s O-D$ pair on path $p$ at time $t ; f_{r s p}(t)$ is the departure flow rate on path $p$ between an $r s O-D$ pair departing at time $t$. Equation (2.6) ensures the FIFO behavior on the running part by forcing vehicles that enter the link at $t$ to be pushed out at $t+d r_{a}(t)$.

The number of vehicles existing on the running part of link $a$ is updated accordingly as given by (2.7) and (2.8):

$$
\begin{gathered}
x r_{r s p}^{a}(t+1)=x r_{r s p}^{a}(t)+u r_{\mathrm{rsp}}^{a}(t)-v r_{r s p}^{a}(t), \quad \forall r, s, a, t, \forall p \in p_{r s} \\
x r_{a}(t)=\sum_{r \in R} \sum_{s \in S} \sum_{\substack{p: a \in p \\
p \in p_{r s}}} x r_{r s p}^{a}(t), \quad \forall a, t
\end{gathered}
$$

where $x r_{r s p}^{a}(t)$ denotes the number of vehicles on the running part of link a of $r s O-D$ pair on path $p$ at time $t$.

\subsection{Node Model}

We only consider nodes with competition. Most node models presented in previous research deal with flow propagation through nodes by distributing demand of upstream links to downstream links. The demand of a link, $D$, is the maximum possible exit flow rate, that is,

$$
D=\min \{C, Q\},
$$

the supply of a link, $S$, is the maximum possible receiving flow rate, that is,

$$
S=\min \{C, R\},
$$

where $C$ is the flow (exit) capacity; $Q$ is the rate of flow ready to exit; $R$ is the maximum entry flow rate to the downstream link permitted by the current traffic condition. The partial flow exit from an upstream link entering a downstream link is calculated according to the turning proportion and constrained by prevailing supply. Taking Figure 1 as an example, the queue on link $a$ (or link $b$ ) is modeled as a whole, time-dependent partial flow exiting from link $a$ to link $c$ (or $d$ ) is calculated as follows: exit flow from link $a$ is determined first with the formula mentioned above, and the partial flow is calculated with the total exit flow from link $a$ multiplying by a turning proportion. To satisfy FIFO rule at link level, such models 


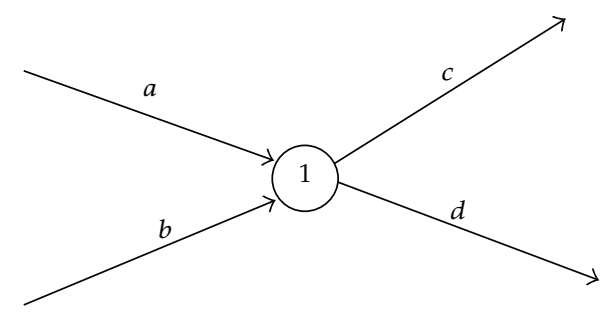

Figure 1: A sample network.

may lead to unnecessary queuing of vehicles. For example, vehicles on link $a$ ready to enter link $c$ will have to wait even if link $c$ has enough supply, just for the reason that link $d$ got a traffic jam and cannot allow any entering flow at current time. In other words, vehicles with different turning directions will have to travel synchronously to satisfy FIFO at link level.

In real traffic conditions, as pointed in HCM, there exist directional turning lanes at intersections especially those with signal controlled, and the capacity of turning lanes rather than that of the whole link actually plays role in limiting flow exiting a link. Drivers generally change or choose turning lanes according to their turning directions when approaching an intersection, which leads to different queues with different flow dynamics in turning lanes. To overcome this shortcoming in describing real dynamics of traffic flow, queuing in different turning lanes should be specifically described, and FIFO at lane level rather than link level should be pursued.

In such situation, the input to the node model is time-dependent entering flow to the queuing part of a link in each turning lane, which can be determined by the exit flow of the running part of the same link according to path flows as given below:

$$
\begin{gathered}
u q_{r s p}^{a a^{+}}(t)=v r_{r s p}^{a, a^{+}}(t), \quad \forall r, s, t, \forall p \in p_{r s}, a, a^{+} \in p, \\
u q_{a a^{+}}(t)=\sum_{r \in R} \sum_{s \in S} \sum_{\substack{p: p \in p_{r s} \\
a, a^{+} \in p}} u q_{r s p}^{a a^{+}}(t), \quad \forall r, s, t, \forall a, a^{+}: a_{h}=a_{t}^{+},
\end{gathered}
$$

where $u q_{r s p}^{a a^{+}}(t)$ is the partial entering flow to the queuing part of link $a$ in turning lane $a a^{+}$of $r s O-D$ pair on path $p$ at time $t$, and $u q_{a a^{+}}(t)$ is the total entering flow to the queuing part of link $a$ in turning lane $a a^{+}$at time $t$.

Based on the entering flow, the exit flow of the directional queuing part can be calculated with prevailing demand and supply as following:

$$
v q_{a a^{+}}(t)=\min \left[S_{a a^{+}}(t), R_{a a^{+}}(t)\right], \quad \forall a, a^{+}: a_{h}=a_{t}^{+},
$$

where $v q_{a a^{+}}(t)$ is the exit flow from the queuing part of link $a$ in turning lane $a a^{+}$at time $t$; $S_{a a^{+}}(t)$ is the partial demand that is present from link $a$ to link $a^{+}$at time $t$ and is determined with (2.14):

$$
S_{a a^{+}}(t)=\left\{\begin{array}{ll}
u q_{a a^{+}}(t), & \text { if } x q_{a a^{+}}(t)=0, u q_{a a^{+}}(t)<c_{a a^{+}}, \\
c_{a a^{+}}, & \text {otherwise, }
\end{array} \quad \forall a, a^{+}: a_{h}=a_{t}^{+},\right.
$$


where $x q_{a a^{+}}(t)$ denotes the number of vehicles on the queuing part of link $a$ in turning lane $a a^{+}$at time $t ; c_{a a^{+}}$denotes the capacity of turning lane $a a^{+}$on link $a$.

The partial supply of link $a^{+}$allocated to link $a$ at time $t$ (denoted with $R_{a a^{+}}(t)$ ) can be calculated by (2.15), as shown below:

$$
\begin{gathered}
R_{a a^{+}}(t)=\beta_{a a^{+}} \cdot R_{a^{+}}(t), \quad \forall a, a^{+}: a_{h}=a_{t}^{+}, \\
R_{a^{+}}(t)=\left\{\begin{array}{ll}
s_{a^{+}}, & \text {if } x_{a^{+}}(t)<h_{a^{+}} \\
v q_{a^{+}}(t), & \text { otherwise }
\end{array} \quad \forall a, a^{+}: a_{h}=a_{t}^{+},\right.
\end{gathered}
$$

where $\beta_{a a^{+}}$is the proportionality coefficient which depends on the lane number and control mode of an intersection and treated as a constant; $h_{a^{+}}$is the maximum number of vehicles that link $a^{+}$can accommodate; $s_{a^{+}}$is the entering capacity of link $a^{+} ; a_{h}$ is the head node of link $a ; a_{t}^{+}$is the tail node of link $a^{+}$.

With partial exit flow of each turning lane known, the total exit flow of the queuing part of link a (denoted with $v q_{a}(t)$ ) can be determined by (2.16) as given below:

$$
v q_{a}(t)=\sum_{a^{+}: a_{h}=a_{t}^{+}} v q_{a a^{+}}(t), \quad \forall a
$$

The relationships between disaggregated and aggregate variables are calculated as follows:

$$
v q_{r s p}^{a a^{+}}(t)=\left\{\begin{array}{ll}
\frac{x q_{r s p}^{a a^{+}}(t)}{x q_{a a^{+}}(t)} \cdot v q_{a a^{+}}(t), & \text { if } x q_{a a^{+}}(t)>0, \\
\frac{u q_{r s p}^{a a^{+}}(t)}{u q_{a a^{+}}(t)} \cdot v q_{a a^{+}}(t), & \text { otherwise. }
\end{array} \quad \forall r, s, t, \forall p \in p_{r s}\right.
$$

The splitting rate $\lambda_{a a^{+}}^{p}$ for the flow that is exiting link $a$ in turning lane $a a^{+}$along path $p$ is calculated as given by (2.18). The constraint associated with $\lambda_{a a^{+}}^{p}$ is given by (2.19), which expresses that at turning lane level, the FIFO rule holds.

$$
\begin{aligned}
& \lambda_{a a^{+}}^{p}=\left\{\begin{array}{ll}
\frac{x q_{\mathrm{rsp}}^{a a^{+}}(t)}{x q_{a a^{+}}(t)}, & \text { if } x q_{a a^{+}}(t)>0, \\
\frac{u q_{\mathrm{rsp}}^{a a^{+}}(t)}{u q_{a a^{+}}(t)}, & \text { otherwise, }
\end{array} \quad p \in p_{r s}, a, a^{+} \in p,\right. \\
& \qquad \sum_{p \in p_{r s}} \lambda_{a a^{+}}^{p}=1 .
\end{aligned}
$$

The number of existing vehicles at time $t$, specified to path, turning lane(s), and queuing part, is updated as follows:

$$
x q_{r s p}^{a a^{+}}(t+1)=x q_{r s p}^{a a^{+}}(t)+u q_{r s p}^{a a^{+}}(t)-v q_{r s p}^{a a^{+}}(t), \quad \forall r, s, t, \forall p \in p_{r s}, a, a^{+} \in p,
$$




$$
\begin{gathered}
x q_{a a^{+}}(t)=\sum_{r \in R} \sum_{s \in S} \sum_{\substack{p: p \in p_{r s} \\
a, a^{+} \in p}} x q_{r s p}^{a a^{+}}(t), \quad \forall a, a^{+}: a_{h}=a_{t}^{+}, \\
x q_{a}(t)=\sum_{a^{+}: a_{h}=a_{t}^{+}} x q_{a a^{+}}(t), \quad \forall a .
\end{gathered}
$$

\section{Solution Procedures}

The solution procedures sought for the DNL problem can be clustered into three approaches: (1) an analytical approach, (2) a simulation-based approach, and (3) a hybridization of these two approaches. The DNL problem solved with an analytical approach possesses the desired properties of both the solution and the link performance function [23]. The simulationbased approach, that is, INTEGRATION, DYSMART, and other propositions, provides more flexibility and consequently enables the reproduction of detailed flow dynamics both in dealing with more complex traffic patterns and in obtaining more realistic loading results lacking the desired solution properties [24]. The hybrid approach set out by the incorporation of an analytical formulation with simulation and utilized in several studies, including [12, $22,25]$ and this paper, has become an efficient alternative in network performance modeling. The feature that it simultaneously possesses both ability of analytical models in obtaining more accurate solutions and the ability of simulation models in obtaining computationally efficient solutions in network traffic modeling context has motivated us to employ a hybrid approach in this paper.

The analysis period is divided into small time intervals with same length $\sigma$. The interval $\sigma$ is treated as unit of time, and time period that is not an exact multiple of $\sigma$ involved in loading procedure is processed with linear approximation. Given path flows during $O-D$ traffic period, we can get entering flow to the running part of each link by (2.6).

The following algorithm summarizes the determination of flows and the number of vehicles on all links by the proposed node-based modeling approach when a network is considered.

(1) Set $t=0$ (first time interval), clear the network, that is,

$$
x r_{r s p}^{a}(t)=0, \quad x q_{r s p}^{a a^{+}}(t)=0, \quad \forall r, s, t, a, p, \forall a^{+}: a_{t}^{+}=a_{h} .
$$

(2) Determine the number of vehicles on the running part and the directional queuing part on each link with (2.8) and (2.22), respectively.

(3) Determine the disaggregate inflow $u r_{r s p}^{a}(t)$ to running parts of links with (2.6).

(4) Calculate the travel time $d r_{a}(t)$ of each link with (2.1)-(2.4) and obtain outflow $v r_{r s p}^{a}\left(t+d r_{a}(t)\right)$ by $(2.5)$.

(5) Calculate the disaggregate number of vehicles $x r_{r s p}^{a}(t)$ with (2.7).

(6) Obtain the disaggregate inflow $u q_{r s p}^{a a^{+}}(t)$ with (2.11).

(7) Calculate the aggregate inflow $u q_{a a^{+}}(t)$ with (2.12) and calculate the number of vehicles in each turning queue $x q_{a a^{+}}(t)$ with (2.21).

(8) Obtain $v q_{a a^{+}}(t)$ and $v q_{a}(t)$ by (2.13)-(2.16). 


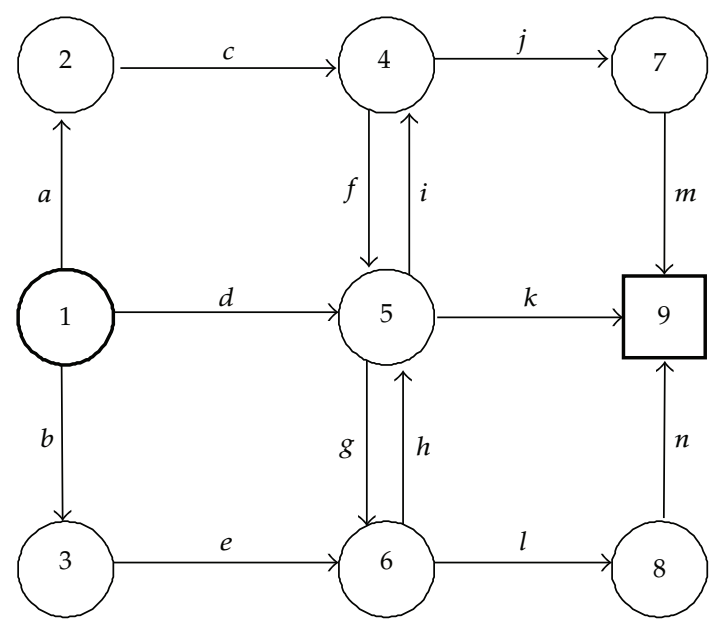

Figure 2: Sample network used for testing trials.

(9) Determine the disaggregate variable $v q_{r s p}^{a a^{+}}(t)$ and $x q_{r s p}^{a a^{+}}(t)$ with (2.17) and (2.20), respectively.

(10) If the demand is discharged from all the paths during the analysis period, stop; otherwise, set $t=$ (following time interval) and go to step (2).

\section{Numerical Implementations}

\subsection{Sample Network}

The performance of the proposed model is tested on a sample network shown in Figure 2 with several paths between the given $O-D$ pair. The link characteristics assigned are given in Table 1, and the characteristics of turning lane(s) at the entrance of each intersection are given in Table 2. The traffic flow interval, $\sigma$, is $1 \mathrm{~min}$, and the departure period, $T$, is $30 \mathrm{~min}$ and is divided into 5 intervals with same length. There is only one $O-D$ pair and seven routes used in this network. Route departure flows, $f_{r s p}(t)$, have a constant value during each departure interval, given in Table 3 and $f_{r s p}(t)=0$ at other times.

\subsection{Test Results}

The performance of the proposed model is evaluated with a number of critical terms from the simulation. The outflow diagram of flow propagation at node 5 is given in Figure 3 . The dynamic outflow and queue length diagrams in each turning lane on link $d$ are given in turn in Figures 4 and 5.

In Figure 3, it is seen that the loadings to link $k$ required longer time, due to a longer flow profile of paths involving this link. It can be seen from Figure 4 that outflows of different turning lanes on link $d$ appear to vary in similar manners, which reach saturation in a few minutes and keep the state for some time (41 minutes in the right turn lane, 52 minutes in the left turn lane, and 69 minutes in the through lane) before a sharp decline. From Figure 5, 
Table 1: Assigned link characteristics of sample network.

\begin{tabular}{lcccccc}
\hline $\begin{array}{l}\text { Link } \\
\text { number }\end{array}$ & $\begin{array}{c}\text { Free-flow speed } \\
v_{f}(\mathrm{~km} / \mathrm{h})\end{array}$ & $\begin{array}{c}\text { Minimum speed } \\
v_{\min }(\mathrm{km} / \mathrm{h})\end{array}$ & $\begin{array}{c}\text { Link length } \\
l(\mathrm{~m})\end{array}$ & $\begin{array}{c}\text { Lane number } \\
\text { lan }\end{array}$ & $\begin{array}{c}\text { Capacity } \\
r c_{a}(\mathrm{pcu} / \mathrm{h})\end{array}$ & $\begin{array}{c}\text { Jam density } \\
k_{j}(\mathrm{pcu} / \mathrm{km} / \mathrm{lan})\end{array}$ \\
\hline$a$ & 30 & 12 & 980 & 2 & 1900 & 140 \\
$b$ & 32 & 10 & 950 & 2 & 1800 & 140 \\
$c$ & 30 & 5 & 800 & 2 & 1700 & 140 \\
$d$ & 36 & 15 & 1750 & 3 & 2600 & 140 \\
$e$ & 30 & 12 & 900 & 2 & 1850 & 140 \\
$f$ & 30 & 10 & 860 & 2 & 1600 & 140 \\
$g$ & 28 & 10 & 750 & 2 & 1850 & 130 \\
$h$ & 30 & 10 & 950 & 2 & 1900 & 130 \\
$i$ & 28 & 13 & 800 & 2 & 1850 & 135 \\
$j$ & 30 & 15 & 830 & 2 & 2000 & 150 \\
$k$ & 35 & 15 & 1250 & 2 & 2050 & 153 \\
$l$ & 30 & 15 & 850 & 2 & 1700 & 160 \\
$m$ & 32 & 14 & 900 & 1 & 1100 & 150 \\
$n$ & 30 & 12 & 880 & 2 & 2200 & 145 \\
\hline
\end{tabular}

Table 2: Capacities of turning lanes at intersection entrance.

\begin{tabular}{lcc}
\hline Link number & Turn direction and downstream link & Capacity $(\mathrm{pcu} / \mathrm{h})$ \\
\hline$a$ & Right turn $\rightarrow c$ & 1500 \\
$b$ & Left turn $\rightarrow e$ & 1600 \\
& Left turn $\rightarrow f$ & 900 \\
Through $\rightarrow j$ & 950 \\
& Right turn $\rightarrow g$ & 900 \\
$d$ & Left turn $\rightarrow i$ & 305 \\
& Through $\rightarrow k$ & 315 \\
& Left turn $\rightarrow h$ & 329 \\
$e$ & Through $\rightarrow l$ & 880 \\
& Left turn $\rightarrow k$ & 265 \\
$f$ & Through $\rightarrow g$ & 507 \\
$g$ & Left turn $\rightarrow l$ & 907 \\
$h$ & Right turn $\rightarrow k$ & 917 \\
$i$ & Through $\rightarrow i$ & 362 \\
$j$ & Right turn $\rightarrow j$ & 1700 \\
$l$ & Right turn $\rightarrow m$ & 1800 \\
\hline
\end{tabular}

Table 3: Route departure flow rates in 30 minutes.

\begin{tabular}{|c|c|c|c|c|c|}
\hline \multicolumn{6}{|c|}{ Departure interval } \\
\hline Route & {$[0,5]$} & {$[6,11]$} & {$[12,17]$} & {$[18,23]$} & {$[24,30]$} \\
\hline (1) $d-k$ & $600(\mathrm{pcu} / \mathrm{h})$ & 720 & 730 & 660 & 0 \\
\hline (2) $d-i-j-m$ & 450 & 540 & 520 & 450 & 0 \\
\hline (3) $b-e-h-k$ & 600 & 720 & 720 & 710 & 550 \\
\hline (4) $b-e-l-n$ & 900 & 1080 & 1080 & 1080 & 870 \\
\hline (5) $d-g-l-n$ & 1090 & 1320 & 1320 & 1130 & 0 \\
\hline (6) $a-c-f-k$ & 410 & 480 & 510 & 510 & 50 \\
\hline (7) $a-c-j-m$ & 1100 & 1320 & 1320 & 1320 & 140 \\
\hline
\end{tabular}




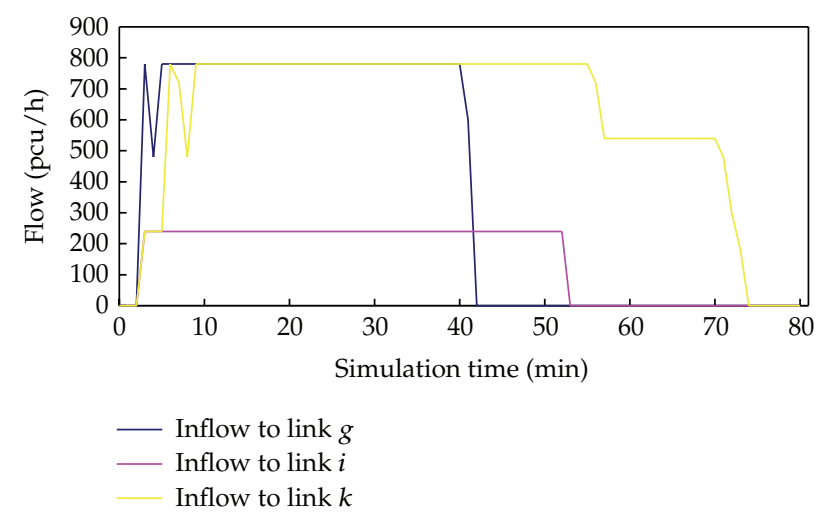

Figure 3: Outflows at node 5 of sample network.

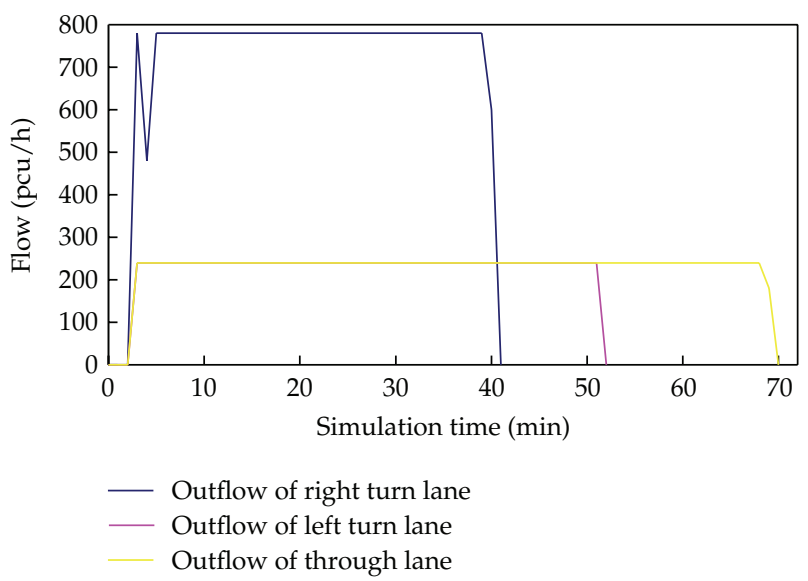

Figure 4: Outflows from turning lane(s) of link $d$.

we can see that the number of queuing vehicles in each turning lane increases to a maximum value after the entrance lanes got saturation.

\subsection{Comparison of Different Models}

In this part, link $d$ was implemented using other models for comparison. Travel-TimeFunction-based link models (TTF) that has been used in [22], node model pursuing FIFO at link level (for simplicity named NM-1) adopted in [18], and node model presented in this paper (for simplicity named NM-2) are tested. In TTF-based link model, a link was treated as a whole, and the travel speed for a vehicle entering link $a$ at time $t$ was calculated with the modified Green-Shields model shown in (2.6). The difference is that the traversal time was calculated with the total length of link $a$, not the partial length of the running part, divided by the speed at time $t$. NM-1 has a similar framework with NM-2, in which a link is divided into two parts and the flow dynamics on running part were described with a travel time function. It is the description of flow dynamics on queuing parts that makes NM-2 different from NM1. In NM-1, time-dependent exit flow of a whole link but not a turning lane is calculated with 


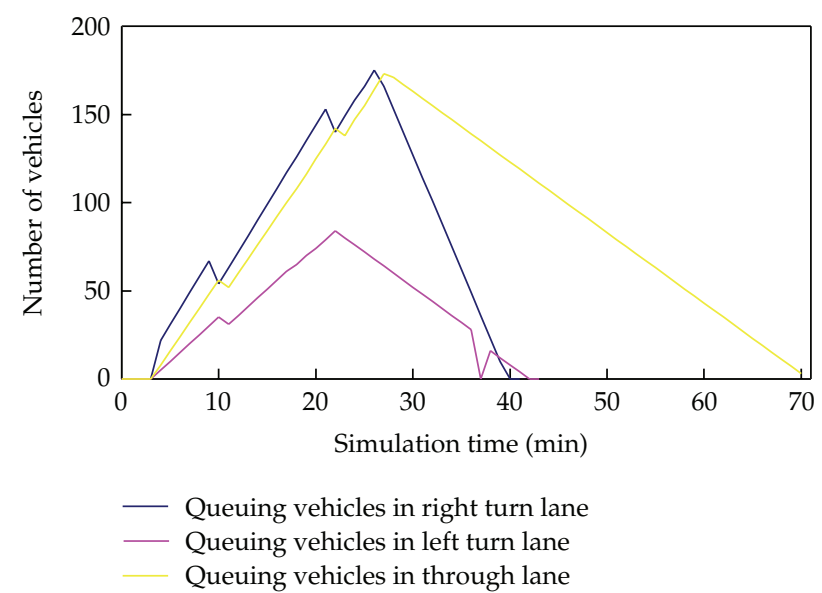

Figure 5: Queue length in turning lane(s) of link $d$.

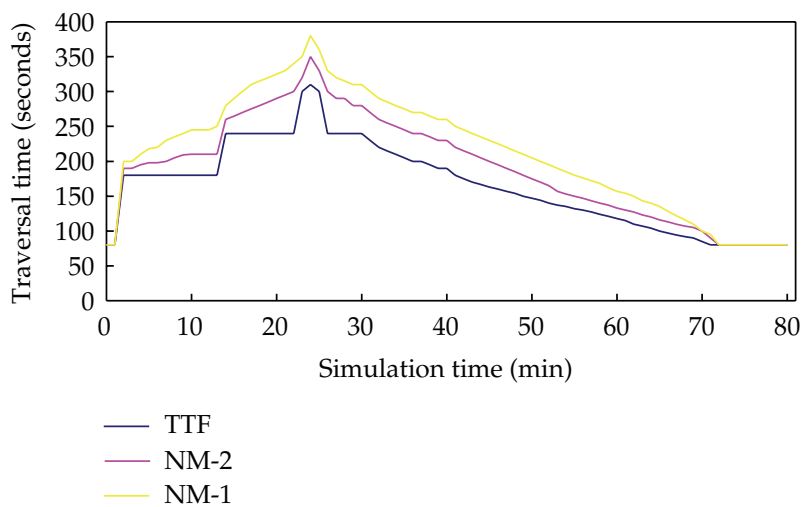

Figure 6: Time-dependent traversal times on link $d$ in different scenarios.

its prevailing demand and supply; queuing vehicles with different directions are described as mixed together; the prevailing demand waiting to exit is limited by the capacity of the link, rather than specified to turning lanes.

Figure 6 compares the time-dependent link traversal times on link $d$ produced by the three models mentioned above. As shown, NM-1 overestimated the congestion on link $d$, while TTF led to a slight underestimation. The reason lies in that the TTF-based link model ignores queuing delay before intersections, and NM-1, pursuing FIFO at link level, may lead to unnecessary queuing of some vehicles.

\section{Conclusion}

In this paper, an analytical node-based model has been proposed for the continuous dynamic network loading problem (CDNLP). Taking account of a set of analytical rules, an algorithm has been derived. The new dynamic node model proposed in this paper is unique in that it explicitly captures the turning lane capacity and directional physical queuing in the description of traffic propagation through nodes. 
In the proposed node model, directional queue in each turning lane is explicitly considered and the FIFO rule at lane level rather than link level is pursued. A directional exit flow function is presented to update the exit flow from each turning lane with the constraints of lane capacity, downstream link capacity, and flow conservation, and so forth. A travel time function (TTF-) based link model has been evolved to a node model in the modeling structure. To capture queue spillback caused by the capacity of turning lanes as well as the downstream link, the concept of prevailing partial length is adopted, which tells the length of partial link that can be used by running vehicles and is determined by the number of running vehicles as well as queuing vehicles. The time to travel the running part of a link equals to the partial length divided by the prevailing speed calculated with a modified Green-shields formulation and is common to vehicles entering the link at the same time. An algorithm is presented for the CDNL problem with our node-based modeling approach, which is based on simulation and updates the performance of running parts as well as queuing parts in order of time step.

The drawback is that the node model integrates a priori mean effects on flows of a traffic signal without explicitly representing the alternation of green and red stages, and traffic wave can not be tracked, for the running part of a link is treated as a homogeneous road segment.

The proposed node-based model can be easily integrated as a flow modeling component of a dynamic traffic-assignment process, enabling its utilization in a wide range of intelligent transportation system applications. For example, the realistic representation of traffic flow dynamics enables the proposed model to be easily utilized in advanced traveler information systems (ATISs). The prediction on link performances can be obtained in terms of realtime flow volume data inputs. These predictions can be basic inputs to ATIS applications, such as variable message signs for route guidance. Dynamic signal optimization and ramp metering are other possible topics that the proposed model's extensions can study for capacity management and speed regulation.

\section{Acknowledgments}

The author would like to thank the supports by the New Teacher Fund for Doctor Station of the Ministry of Education with no. 20116204120005, by Program of Humanities and Social Science of Education Ministry of China for Western and Frontier under Grant no. 12XJCZH002 and 11XJC630009.

\section{References}

[1] J. H. Wu, Y. Chen, and M. Florian, "The continuous dynamic network loading problem: a mathematical formulation and solution method," Transportation Research Part B, vol. 32, no. 3, pp. 173-187, 1998.

[2] Y. W. Xu, J. H. Wu, M. Florian, P. Marcotte, and D. L. Zhu, "Advances in the continuous dynamic network loading problem," Transportation Science, vol. 33, no. 4, pp. 341-353, 1999.

[3] M. Blumberg and H. Bar-Gera, "Consistent node arrival order in dynamic network loading models," Transportation Research Part B, vol. 43, no. 3, pp. 285-300, 2009.

[4] J. M. Rubio-Ardanaz, J. H. Wu, and M. Florian, "Two improved numerical algorithms for the continuous dynamic network loading problem," Transportation Research Part B, vol. 37, no. 2, pp. 171-190, 2003. 
[5] V. Astarita, "A continuous time link model for dynamic network loading based on travel time function," in Proceedings of the 13th International Symposium on Transportation and Traffic Theory, pp. 79-102, Lyon, France, 1996.

[6] H. Bar-Gera, "Continuous and discrete trajectory models for dynamic traffic assignment," Networks and Spatial Economics, vol. 5, no. 1, pp. 41-70, 2005.

[7] R. Jayakrishnan, H. S. Mahmassani, and T. Y. Hu, "An evaluation tool for advanced traffic information and management systems in urban networks," Transportation Research Part C, vol. 2, no. 3, pp. 129-147, 1994.

[8] Q. Yang and H. N. Koutsopoulos, "A microscopic traffic simulator for evaluation of dynamic traffic management systems," Transportation Research Part C, vol. 4, no. 3, pp. 113-129, 1996.

[9] V. Astarita, M. Florian, M. Mahut, and J. H. Wu, "Modeling the spill-back of congestion in link based dynamic network lloading models: a simulation model with application," in Proceedings of the 14th International Symposium on Transportation and Traffic Theory, pp. 55-573, Jerusalem, 1999.

[10] M. Carey and Y. E. Ge, "Convergence of a discretised travel-time model," Transportation Science, vol. 39, no. 1, pp. 25-38, 2005.

[11] X. Nie and H. M. Zhang, "A comparative study of some macroscopic link models used in dynamic traffic assignment," Networks and Spatial Economics, vol. 5, no. 1, pp. 89-115, 2005.

[12] C. F. Daganzo, "The cell transmission model, part II: network traffic," Transportation Research Part B, vol. 29, no. 2, pp. 79-93, 1995.

[13] D. K. Merchant and G. L. Nemhauser, "Model and an algorithm for the dynamic traffic assignment problems," Transportation Science, vol. 12, no. 3, pp. 183-199, 1978.

[14] C. F. Daganzo, "Properties of link travel time functions under dynamic loads," Transportation Research Part B, vol. 29, no. 2, pp. 95-98, 1995.

[15] J. P. Lebacque, "The Godunov scheme and what it means for first order traffic flow models," in Proceedings of the 13th International Symposium on Transportation and Traffic Theory, pp. 647-677, Lyon, France, 1996.

[16] S. Mammar, J. P. Lebacque, and H. Haj Salem, "Intersection modeling based on the second order traffic flow model (ARZ)," in Proceedings of the 11th EWGT Meeting, pp. 435-440, 2006.

[17] H. B. Celikoglu, "A dynamic node model with a quadratic travel time function based link model," in Proceedings of the 11th EWGT Meeting, pp. 658-666, 2006.

[18] H. B. Celikoglu, E. Gedizlioglu, and M. Dell'Orco, "A node-based modeling approach for the continuous dynamic network loading problem," IEEE Transactions on Intelligent Transportation Systems, vol. 10, no. 1, pp. 165-174, 2009.

[19] H. M. Zhang and Y. Nie, "Modeling network flow with and without link interaction: properties and implications," in Proceedings of the 84th annual meeting of the Transportation Research Board, Washington, DC, USA, 2006.

[20] M. Carey and M. McCartney, "Behaviour of a whole-link travel time model used in dynamic traffic assignment," Transportation Research Part B, vol. 36, no. 1, pp. 83-95, 2002.

[21] C. F. Daganzo, "A variational formulation of kinematic waves: solution methods," Transportation Research Part B, vol. 39, no. 10, pp. 934-950, 2005.

[22] R. Jayakrishnan, W. K. Tsai, and A. Chen, "A dynamic traffic assignment model with traffic-flow relationships," Transportation Research Part C, vol. 3, no. 1, pp. 51-72, 1995.

[23] B. W. Wie, R. L. Tobin, and M. Carey, "The existence, uniqueness and computation of an arc-based dynamic network user equilibrium formulation," Transportation Research Part B, vol. 36, no. 10, pp. 897-918, 2002.

[24] H. B. Celikoglu and M. Dell'Orco, "Mesoscopic simulation of a dynamic link loading process," Transportation Research Part C, vol. 15, no. 5, pp. 329-344, 2007.

[25] A. K. Ziliaskopoulos, "Linear programming model for the single destination System Optimum Dynamic Traffic Assignment problem," Transportation Science, vol. 34, no. 1, pp. 37-49, 2000. 


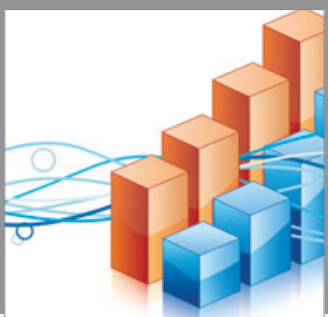

Advances in

Operations Research

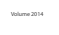

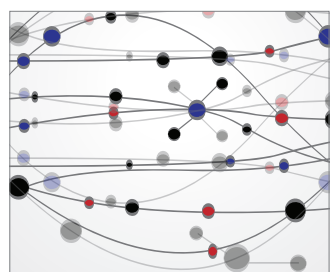

\section{The Scientific} World Journal
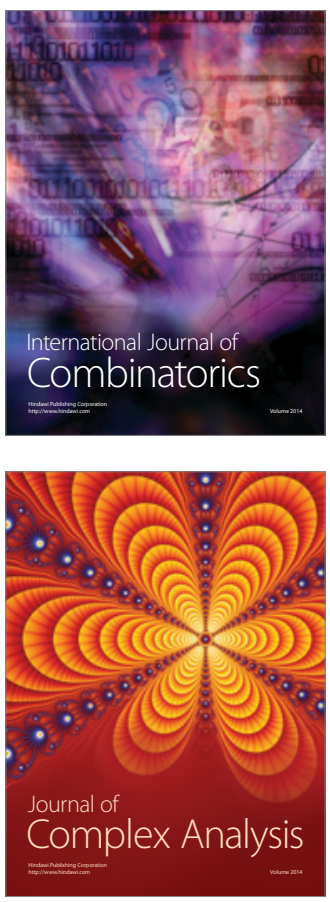

International Journal of

Mathematics and

Mathematical

Sciences
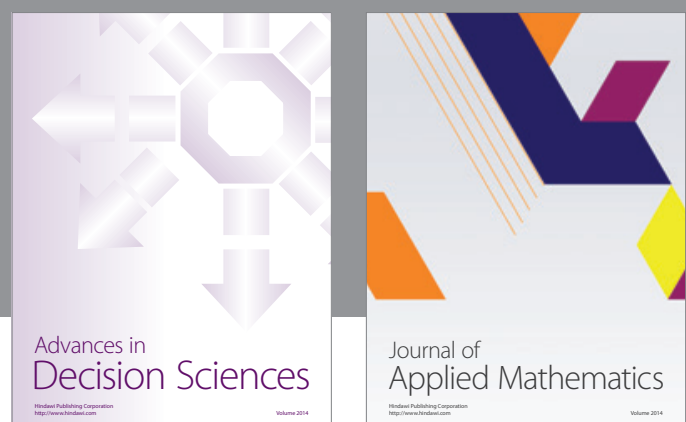

Journal of

Applied Mathematics
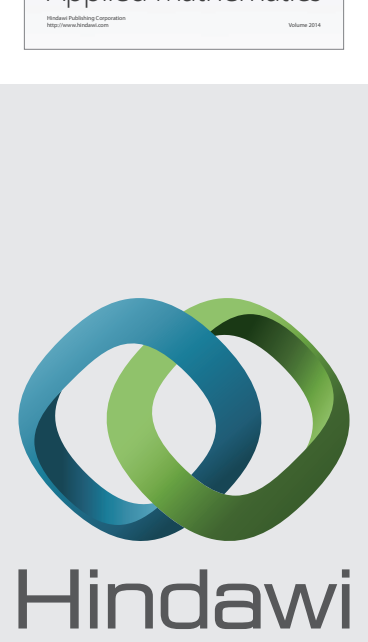

Submit your manuscripts at http://www.hindawi.com
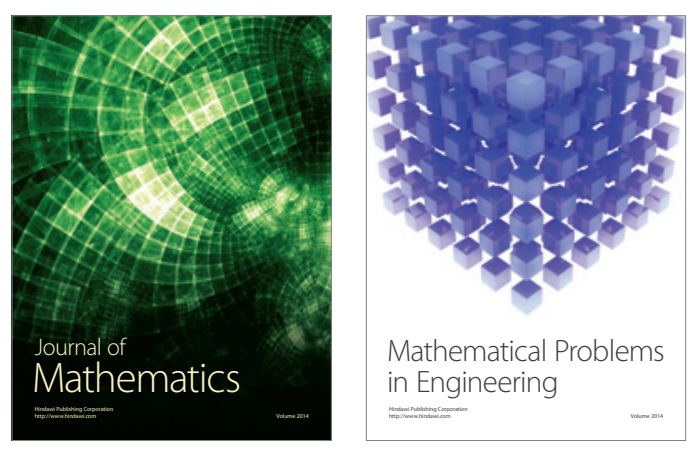

Mathematical Problems in Engineering
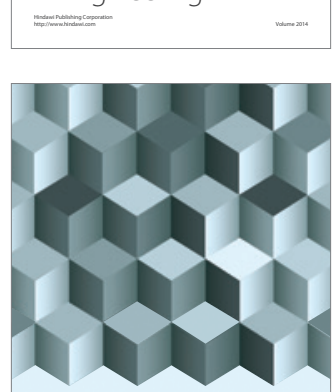

Journal of

Function Spaces
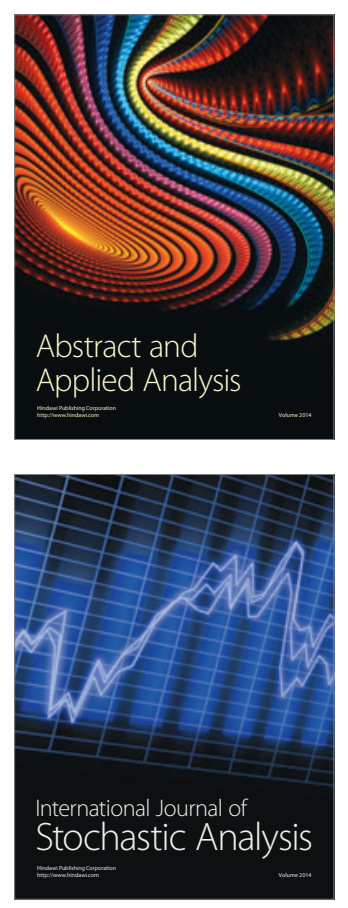

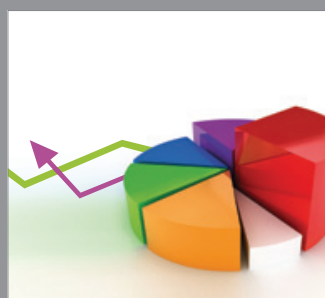

ournal of

Probability and Statistics

Promensencen
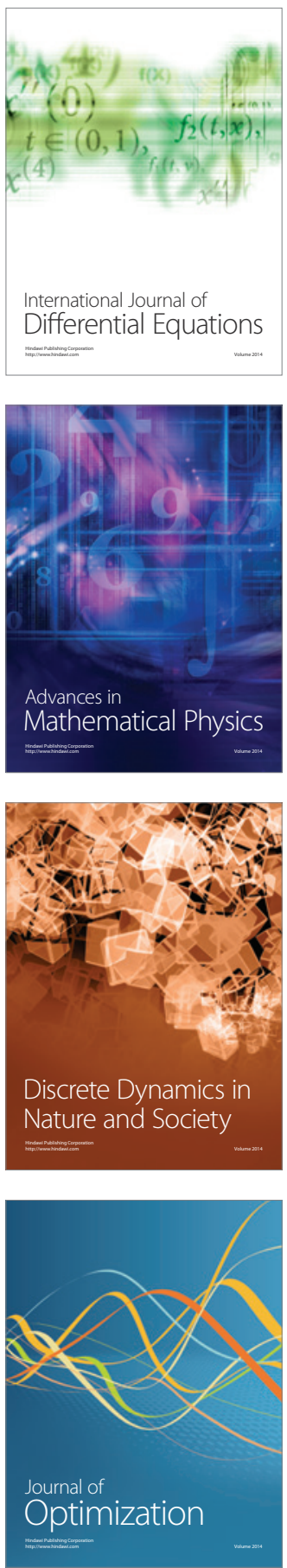